\title{
Community mental health care after self harm: A retrospective cohort study
}

\section{Authors and affiliations:}

\author{
Matthew J. Spittal ${ }^{1}$ \\ Fiona Shand ${ }^{2}$ \\ Helen Christensen ${ }^{2}$ \\ Lisa Brophy $^{1}$ \\ Jane Pirkis ${ }^{1}$
}

1. Melbourne School of Population and Global Health, The University of Melbourne

2. Black Dog Institute, University of New South Wales

\section{Corresponding author:}

Matthew J. Spittal, Melbourne School of Population and Global Health, The University of Melbourne, Parkville 3010, Victoria, Australia, +61 390358230 (phone), +61 393481174 (fax), m.spittal@unimelb.edu.au (email).

\section{Sources of funding:}

This study was funded by the National Mental Health Commission. The funders had no role in the design and conduct of the study, the collection, analysis, interpretation of the results or preparation of the manuscript.

\section{Author contributions:}

All authors were actively involved in conceptualising the initial research plan. MJS and FS sought access to the data. MJS and JP discussed and decided on the analysis plan. MJS undertook data-management and analysis. All authors contributed to the discussion of findings and the interpretation. MJS prepared the first draft of the manuscript, and revised the manuscript on the basis of comments from the other authors. All authors approved the final version of the manuscript.

\section{Word count:}

256 (abstract); 4,422 (manuscript).

\section{Keywords:}

Suicide; Community mental health care; Epidemiology; Mental Health 


\section{Abstract}

\section{Objective}

Presentation to hospital after self harm is an opportunity to treat underlying mental health problems. We aimed to describe the pattern of mental health contacts following hospital admission focusing on those with and without recent contact with community mental health services (connected and unconnected patients).

\section{Methods}

We undertook a data linkage study of all individuals admitted as a general or psychiatric inpatient to hospital after self harm in New South Wales, Australia, between 2005 and 2011. We identified the proportion of admissions where the patient received subsequent in-person community mental health care within 30 days of discharge and the factors associated with receipt of that care.

\section{Results}

A total of 42,891 individuals were admitted to hospital for self harm. In $41 \%$ of admissions the patient had contact with a community mental health service after discharge. Patients connected with community mental health services had 5.33 (95\% CI 5.09 to 5.59) times higher odds of follow-up care than unconnected patients. Other factors, such as increasing age and treatment as a psychiatric inpatient were associated with lower odds of follow-up community care.

\section{Conclusions}

Our study suggests that full advantage is not being taken of the opportunity to provide comprehensive mental health care for people who self harm once they have been discharged from the inpatient setting. This is particularly the case for those who have not previously received community mental health care. There appears to be scope for system-level improvement in the way in which those who are treated for self harm are followed up in the community. 


\section{Introduction}

Mental illness is consistently identified as one of the most significant risk factors for self harm and suicide (Harris and Barraclough, 1997; Haw et al., 2001; Singhal et al., 2014). Some individuals who self harm have long standing histories of mental health service use, and others have had no previous contact with the mental health system (Hawton et al., 2012; Murphy et al., 2011). Either way, hospital admissions for self harm represent opportunities for the assessment and treatment of underlying mental health problems in the hospital setting, and for linking patients into ongoing community-based care.

To date, little research has examined mental health care provision following admission to hospital for self harm. Most of the studies that have been done have focused on emergency department presentations, rather than admissions. For example, studies from the US (Olfson et al., 2012), the UK (Bennewith et al., 2004; Cooper et al., 2013; Gunnell et al., 2005) and Finland (Suominen et al., 2004; 2002) suggest that fewer than half of all patients presenting to the emergency department for self harm are admitted to hospital, about one-tenth are admitted to a psychiatric ward, and at least half do not receive follow-up mental health treatment after discharge. Olfson and colleagues (2012) found that decisions to admit patients to hospital were related to some known risk factors for suicide (age, lethality of the self harm method and previous mental health treatment) but unrelated to others (prior self harm events, diagnosis of depression, schizophrenia or substance use disorder). These findings are important, but they do not shed light on what happens for those who are admitted to hospital, who arguably, may be at greatest risk of suicide after discharge, especially in the first four weeks (Carroll et al., 2014; Gunnell et al., 2008).

We conducted a data linkage study to explore the provision of community mental health services for patients discharged from public hospitals after self harm. Our focus is on services that involve in-person clinical contact (as opposed to services performed on behalf of patients, such as updating patient notes, organising referrals, etc.). We focused on the use of services within 30 days of discharge and examined this among two groups - those with a recent history of contact with community mental health services at the time there were admitted to hospital (“connected” patients), and those with no such recent history ("unconnected” patients). We deliberately made a distinction between these two groups because we were interested in the extent to which advantage was being taken of the opportunity to link individuals who self harm into ongoing mental health care. We tested several hypotheses. Our first was that after discharge for self harm, rates of community mental health care would be low. Our second was that after discharge, those patients already connected to community mental health services would be more likely to receive community mental health care than patients who were unconnected. Our third was that some groups would be more likely to receive community mental health care than others, for instance, psychiatric inpatients, patients who used lethal methods of self harm, patients with a psychiatric diagnosis. 


\section{Method}

\section{Data sources}

We used data from the New South Wales (NSW) health system. NSW is typical of other states and territories in Australia in that its public-sector specialised mental health system is organised into area mental health services. Each area mental health service is funded by the state government and provides inpatient and community care to people with severe mental illness. Inpatient services are largely provided through mental health wards in general hospitals, and community care is provided by teams with a range of foci (e.g., crisis assessment and treatment, longer-term support). Because our data sources relate only to services funded by the state government, we do not have access to information on services funded by the federal government, for example, treatment by general practitioners and psychiatrists and psychologists in private practice.

The Centre for Health Record Linkage (CHeReL) provided us with linked data on inpatient and community mental health service use by people who had self harmed. CHeReL maintains a data linkage system that enables health records from a number of administrative health datasets from NSW to be linked together at the individual level. This includes inpatient data from the NSW Admitted Patient Data Collection, data on contacts with community mental health services from the NSW Mental Health Ambulatory Data Collection, and mortality data from the NSW Register of Births, Deaths and Marriages. Because of changes in the reporting of mental health contact data prior to 2005 and because of the different end dates for each data source, we restricted our analysis to those events occurring between January 2005 and December 2011 (although we use inpatient data prior to this date to construct one variable, admission to hospital for self-harm in the two years prior). A unique person-identifier allowed us to observe the dates of admission and community contact in sequence. Approval for this study was granted by the NSW Population and Health Services Research Ethics Committee.

\section{Procedure}

We linked data on public hospital inpatient admissions for intentional self harm (ICD-10 codes X60-X84) to data on contacts with community mental health services. Individuals could potentially have multiple admissions to hospital for self-harm over the study period; however, we focus on just their index admission (the first admission after January 2005). Our outcome variable was any new in-person contact with community mental health services within 30 days of discharge from hospital. We excluded patients from the analysis who died while an inpatient or within 30 days of discharge.

\section{Variables}

We constructed variables representing the patient's status during their admission. The key variable was a binary variable representing connection with a community mental health service at the time of admission to hospital for self harm (defined as within the study period, having face-to-face contact with community mental health services at least once in the 12 months prior to their admission to hospital for self harm). Variables were also constructed for length of admission ( $\leq 1$ day, 2-4, 5-9, 10-19, 20-29, $\geq 30$ days), admission as a psychiatric inpatient (yes or no), age at admission (15-19, 20-29, 30-39, 40-49, 50-59, $\geq 60$ years), sex, and relationship status at admission (single, in a relationship, unknown), admission for self harm in the previous two years 
(including any admissions prior to January 2005 and coded yes or no), method of self harm at admission, and the presence of seven psychiatric disorders at each admission. Method of self harm was coded into five categories, based on the ICD-10 external cause of injury: poisoning (X60-X66, X68, X69), motor vehicle exhaust gas (X67), hanging (X70), cutting/piercing (X78, X79), jumping (X80), and all other methods (X71X77, X81-X84). Psychiatric disorders were coded into seven categories based on the ICD-10 diagnosis codes (patients could be assigned multiple diagnoses). These were: substance misuse disorder (F10-F19), schizophrenia and related diagnoses (F20-F29), mania (F30, F31), depression, (F32-F39), anxiety (F40-F49), eating disorder (F51-F59), and personality disorders (F51-F59).

\section{Statistical analyses}

We computed descriptive statistics for all variables at admission and a cross-tabulation of treatment by community mental health services within 30 days of discharge by prior contact with community mental health services. We estimated the odds of being treated by community mental health services within 30 days of discharge from hospital using logistic regression. Our model included all patient and admission variables described above. We also undertook two sensitivity analyses. In the first, we excluded all patients from the denominator who were re-admitted to hospital within 30 days and re-estimated our model. In the second, we stratified our analysis by connected/unconnected patients. All analyses were undertaken in Stata 13.1.

(StataCorp, 2013) 


\section{Results}

\section{Patient and admission characteristics}

There were 42,891 individuals admitted to hospital after self harm in NSW between January 2005 and December 2011. A total of 397 died while in hospital and a further 141 died within 30 days of discharge from hospital. Both these groups were excluded from the analysis leaving a remaining sample of 42,353 patients. Their characteristics are shown in Table 1. The majority of admission were for females (57\%), for people who were single (68\%) and for people aged $<40$ years (63\%). Seventy-four percent of patients had a diagnosed psychiatric disorder. The most common diagnoses at admission were depression (37\%), substance misuse disorder (29\%) and anxiety disorder (24\%). The most common method of self harm at admission was poisoning (76\%), followed by cutting/piercing (16\%). The typical length of stay in hospital was 1 day or less (60\%). Seven percent of admissions were to a psychiatric inpatient unit.

\section{Community mental health care after discharge}

In $41 \%$ of inpatient admissions, the patient received follow-up care from a community mental health service within 30 days of discharge from hospital. Among patients unconnected with a community mental health service, this figure was $27 \%$, and among connected patients it was 66\% (Table 2).

\section{Multivariate analysis}

Being connected with a community mental health service was associated with increased odds of receiving community mental health care after discharge from hospital. Those who were already connected with community mental health services had 5.33 times higher odds of having a contact with community mental health services following discharge compared with those who were unconnected with such services (Table 3).

In comparison to patients who stayed in hospital for up to one day (the reference category), lengths of stay in hospital that were between 2 and 9 days were associated with lower odds community mental care following discharge ( $\mathrm{OR}=0.83$ and 0.81 ); lengths of stay longer then this had approximately the same odds as the reference category $(\mathrm{OR}=0.91,1.01$ and 0.87$)$. Patients with an admission to hospital for self harm in the two years prior to their index admission had lower odds of receiving community mental health care $(\mathrm{OR}=0.91)$ compared to those with no previous admissions. Inpatient admission as a psychiatric patient was associated with lower odds of access to community mental health care following discharge ( $\mathrm{OR}=0.88)$, compared to those admitted as general inpatients.

The odds of receiving community mental health care broadly declined with age. Compared to the odds of patients aged 15-19 (the reference category), all other age groups had lower odds (OR range 0.70 to 0.84 ) of community mental health care within 30 days of discharge. Females had 7\% higher odds of receiving community mental health care than males but being single was not associated with access to community mental health care.

The odds of receiving community mental health care also varied by method of self harm. Jumping from heights $(\mathrm{OR}=0.71)$ and the 'other' methods $(\mathrm{OR}=0.82)$ were associated with decreased odds of receiving community care (in comparison to the odds for poisoning). For hanging and motor vehicle exhaust gas, there 
was evidence of increased odds ( $\mathrm{OR}=1.27$ and 1.30). Cutting/piercing had approximately the same odds as the reference category.

A diagnosis of substance misuse disorder (in comparison to those without this diagnosis) was associated with lower odds of receiving community care within 30 days of discharge (OR $=0.78)$. Diagnoses of schizophrenia and related psychoses $(\mathrm{OR}=1.46)$, depression $(\mathrm{OR}=1.24)$ and anxiety $(\mathrm{OR}=1.15)$ were each associated with higher odds of receiving community mental health care (in comparison to those patients without a diagnosis of each of these respective disorders). A personality disorder was associated with lower odds (OR = 0.90). Mania, and eating disorder and were both unrelated to receiving community mental health care.

\section{Sensitivity analysis}

We identified 2,270 cases where the patient was re-admitted to hospital within 30 days. We repeated the analysis excluding these. For all variables except one, this had a negligible effect on the results (see Table S1, supplementary appendix). For example, for a patient already connected with community mental health services, the odds of receiving community care after discharge was 5.59 times higher than for an unconnected patient (95\% CI 5.32 to 5.86). The variable that differed was admission to hospital for self-harm in the previous two years. Those who had a prior admission had 2.22 time higher odds (95\% CI 1.89 to 2.61) of receiving community mental health care compared to those with no prior admission.

We also stratified our analysis by whether or not the patient was connected to community mental health services. Again, differences between the stratified results and main results were small (Table S2, supplementary appendix), with the exceptions that the presence of an eating disorder was associated with lower odds of receiving community care in connected patients $(\mathrm{OR}=0.68,95 \% \mathrm{CI} 0.72$ to 0.90$)$ and that the presence of an anxiety disorder was not associated with community mental health care in connected patients $(\mathrm{OR}=1.02,95 \%$ CI 0.82 to 1.04$)$. 


\section{Discussion}

In this study of individuals discharged from hospital after self harm, we found that $41 \%$ had face-to-face contact with a community mental health services within 30 days of discharge. It is unclear what care, if any, the remaining 59\% received.

\section{Community mental health care following a hospital admission for self harm: Current and ideal practice}

People who self harm often have underlying mental health problems and are at high risk of further self harm and suicide (Hawton and van Heeringen, 2009; Kapur et al., 2015; Nock et al., 2008; Carter et al., 2016; Schaffer et al., 2016; Steeg et al., 2012). Presentation to hospital for treatment of self-inflicted injuries or poisoning therefore presents an opportunity to initiate mental health treatment in hospital and to create pathways for ongoing care in the community (Larkin and Beautrais, 2010).

Our findings suggest that full advantage of this suicide prevention opportunity is not being taken. Only $41 \%$ of those treated as an inpatient had a contact with a community mental health service within 30 days of discharge from hospital. Those who were not already known to the mental health system were particularly disadvantaged; their counterparts who already had some connection to the system had over five times higher odds of having contact with community mental health services post-discharge.

These relatively low levels of community-based follow-up run contrary to clinical practice guidelines for the treatment of self harm from around the world. These guidelines typically recommend that patient management plans should be developed while the patient is in hospital to ensure that community mental health care is made available to him or her shortly after discharge (American Psychiatric Association, 2003; National Institute for Health and Clinical Excellence, 2004; Carter at al., 2016).

\section{Potential factors influencing current practice}

The reasons for these relatively low levels of community follow-up are unknown, but may include a mix of system- and provider-level factors and patient- and carer-level factors. The system- and provider-level factors may relate to insufficient integration between inpatient and community mental health services, and sub-optimal communication between providers in these settings. The area mental health service structure is explicitly designed to provide a smooth pathway through elements of the system, allowing patients to receive the right care in the right place at the right time. Communication between staff working in these system elements may not always work as it is intended to, however, and inpatients may not always be discharged to community care. Arguably, this may be exacerbated in cases where patients are admitted for self harm, particularly if they have not had contact with mental health services in the past. There is evidence from overseas that discussion between inpatient staff and community-based staff about patients' discharge plans improves the likelihood that they will receive community care (Boyer et al., 2000), and putting clear protocols in place and fostering strong collaboration between hospital staff and community mental health teams can reduce subsequent incidents of self harm (Mehlum et al., 2010).

Patient- and carer-level factors may be many and varied. Patients may be reluctant to follow through with a referral to a community mental health service if they feel that they have insufficient control over their own treatment, if they are uncertain about what their ongoing care will involve or if they have been treated poorly by hospital staff. Carers may be disinclined to support patients in transitioning from inpatient to community care if 
they feel sidelined in the process. Again, international studies point to interventions that may be useful here. For example, opportunities for patients to begin their community care program prior to inpatient discharge and family involvement during the hospital stay have both been shown to increase the likelihood of patients attending community mental health service appointments (Boyer et al., 2000). Similarly, mobile support and treatment teams that visit patients in their own environment have been shown to improve the likelihood of an initial community mental health care contact occurring compared with teams located in outpatient settings (Currier et al., 2010).

\section{Lack of connection with mental health services and other risk factors for non-receipt of community mental health care post-discharge}

In considering interventions to encourage referral to and uptake of community mental health care after an admission for self harm, particular attention should be given to patients who have not already been seen by community-based services. The fact that they are not known to community team members may reduce the chances of good communication and planning around their future care. Similarly, the fact that they are not familiar with these services may exacerbate their feelings of alienation and uncertainty.

Some of the other factors that we found to be predictive of non-receipt of community mental health care in our regression analysis are also worthy of comment. There is evidence that contextual factors play a role, but further work is needed to tease out the mechanisms by which these risk factors operate:

- Patients who remain as inpatients for relatively longer periods of time are less likely to receive community mental health care than those with shorter inpatient stays. This is possibly because there is a view that their care has wholly and solely been provided in the hospital setting. Alternatively, those with longer length of stay may be involuntary patients who are subsequently reluctant to engage with followup services. Regardless, if length of stay is taken as a proxy for severity, as it often is, then these patients may well be ones who would particularly benefit from ongoing community care.

- There is some evidence that patients who have had previous admissions for self harm are no more likely to receive community care than those with no history of self harm (although we note that in our sensitivity analysis, a history of self harm was associated with an increase in the likelihood of receiving care). One possibility is that hospital staff see them as being motivated by reasons other than a wish to die (e.g., seeking attention). Another possibility is that the needs of this group may not be considered appropriate, or too difficult to treat, in the community mental health setting (which may not offer long term individual counselling). Nonetheless, repeated self harm confers a high risk for suicide (Owens et al., 2002), and even if these individuals are acting with some degree of ambivalence, it is likely that community mental health care is still warranted.

- Being male and being older reduces the odds of a patient receiving community mental health care. This is of concern because, like a history of self harm, both of these are important risk factors for suicide.

Thought may need to be given to the care pathway between inpatient and community settings, and how it might be better tailored to these demographic groups.

Particular methods of self harm are associated with a diminished likelihood of subsequent community mental health care. It is possible to speculate that the focus in these cases may be on the more physical aspects of care and that immediate and ongoing mental health care may 'take a back seat'. 
- Individuals with substance misuse disorder are comparatively unlikely to receive community mental health care following discharge. In Australia, mental health services and substance use services still operate relatively independently of each other, despite recognition of the high degree of comorbidity between mental disorders and drug and alcohol problems and despite attempts to better align these service sectors. People with substance use disorders may face particular barriers to accessing community mental health care, particularly if their substance use issues are overlaid with a recent history of self harm. Clinical staff may therefore give priority to referring this group to alcohol and drug services over mental health services.

- Our study does not examine suicide as an outcome, but previous research has found that those who received intensive follow-up care in the community after discharge as a psychiatric inpatient were at higher risk of suicide in the year following than those who received less intensive follow-up (Large et al., 2011). This is likely because those identified as being at high risk of suicide while in hospital were then provided with more follow-up care to mitigate that risk.

\section{Strengths and limitations}

Our study has a number of advantages over previous studies because it used statewide linked data which gave us a comprehensive picture of all inpatient admissions for self harm and all subsequent related community mental health care contacts over our study period. In addition, because these data were linked to death records, we were able to reliably exclude from our denominator patients who died in hospital or within 30 days of discharge from hospital.

Our study also has several limitations. Our focus on community mental health care contacts means we may have underestimated the extent to which people admitted to hospital for self harm may receive care postdischarge. This is because a proportion of those who had no subsequent contact with community mental health services in our study may have received ongoing care from other sources. Discharge planning, for instance, would usually include referrals to the patient's general practitioner, psychiatrist or psychologist for further care if they were already in a therapeutic relationship with one of these providers. The service contact datasets we had available to us related to public sector services only. It is possible that some of those who did not receive care from community mental health teams did so from elsewhere (e.g., from primary care such as a general practitioner, psychologist or psychiatrist operating in private practice, or as a private hospital inpatient or outpatient). Given that there is a direct relationship between inpatient and community services within area mental health services (due to shared funding arrangements) (Flannery et al., 2011), it would seem reasonable to expect the latter to be a more common referral destination than our data suggest they are.

The second limitation may have biased the data in the opposite direction. Because we could not directly track referrals to community mental health services, we relied on the occurrence of community mental health contacts within 30 days as evidence of a referral. Some of these contacts may have been unrelated to the hospital admission for self harm, thus inflating the numbers of admissions that were followed by seemingly appropriate care in the community.

Third, it is possible that the relationship between being a connected patient and community mental health care is due to confounding by indication. That is, being a connected patient is a marker for poor mental health, and poor mental health is associated with receiving community mental health care. We were not able to test this 
explanation directly, although our main analysis includes a large number of variables that potentially lie on the causal pathway. We were also able to stratify the analysis by whether or not the patient was connected, and the results from this analysis were similar to the main results. Our effect size was large; nonetheless, the possibility remains that the finding we observe is due to residual confounding.

Fourth, we did not have information on the availability of community mental health services in each area. It is possible that some patients did not access community mental health services - and indeed were admitted to hospital - because community-based services were unavailable to them (see for instance, Burns et al., 2007).

There are several other limitations too, most of which relate to the fact that the breadth of coverage of our data came at the expense of depth. For example, although we were able to examine at a general level what happened during and after admissions to hospital for self harm, we were not able to explore what happened before these admissions. Many of the admissions would have arisen from a presentation to the emergency department, but reliable data on emergency department presentations for self harm is unavailable in Australia (external cause of injury codes, needed to identify cases of self harm, are not recorded in any state or territory emergency department register). Similarly, we did not have the capacity to look at the specific care that was provided to patients, in either the inpatient or community setting. Finally, we did not have access to data on whether patients were being treated under involuntary orders, including whether their admission was an involuntary treatment order or whether they were on a community treatment order prior to their admission or discharge. This may have impacted on their willingness to be in contact with community mental health services and the likelihood of follow-up.

\section{Conclusions}

Effective mental health treatment has the potential to improve outcomes for those who have self harmed, and reduce the likelihood of their engaging in future self harming behaviour (Krysinska, et al., 2016). The period immediately following discharge is particularly important; risk of repeated self harm is at its height in the first four weeks after discharge (Gunnell et al., 2008). Our study suggests that full advantage is not being taken of the opportunity to provide comprehensive specialist mental health care for people who self harm once they have been discharged from the inpatient setting. This is particularly the case for those who have not previously received community mental health care. There would appear to be scope for system-level improvement in the way in which those who are admitted to hospital for self harm are followed up in the community. Ideally, the linkages between inpatient units and community mental health care services should be strengthened so that aftercare becomes more routine and so that it can be monitored (to make sure that care has taken place as planned). Finally, this study highlights that there is scope to improve the linkages between state/territory and Commonwealth data to better understand how mental health services are used (i.e. where patients have been treated by their GP or by a psychologist or psychiatrist in private practice). 


\section{Acknowledgements:}

We thank the Centre for Health Record Linkage in NSW for providing access to the data and staff from the Mental Health and Drug and Alcohol Office at the Ministry of Health for advice on interpreting the results.

\section{Conflicts of interest:}

The authors declare there is no conflicts of interest. 


\section{References}

American Psychiatric Association (2003) Practice guideline for the assessment and treatment of patients with suicidal behaviors. American Journal of Psychiatry 160(11 Suppl): 1-60.

Bennewith O, Gunnell D, Peters T, et al. (2004) Variations in the hospital management of self harm in adults in England: observational study. British Medical Journal, 328(7448): 1108-1109.

Boyer CA, McAlpine DD, Pottick KJ, et al. (2000) Identifying risk factors and key strategies in linkage to outpatient psychiatric care. American Journal of Psychiatry 157(10): 1592-1598.

Burns T, Catty J, Dash M, et al. (2007) Use of intensive case management to reduce time in hospital in people with severe mental illness: systematic review and meta-regression. British Medical Journal, 335(7615): 336.

Carroll R, Metcalfe C and Gunnell D (2014) Hospital Presenting Self-Harm and Risk of Fatal and Non-Fatal Repetition: Systematic Review and Meta-Analysis. PLoS ONE, 9(2): e89944.

Carter, G., Page, A., Large, M., Hetrick, S., Milner, A. J., Bendit, N., Walton, C., Draper, B., Hazell, P., Fortune, S., Burns, J., Patton, G., Lawrence, M., Dadd, L., Robinson, J., Christensen, H. (2016) Royal Australian College of Psychiatrists clinical practice guidelines for the management of deliberate self-harm. Australian and New Zealand Journal of Psychiatry, 50 (10), 939-100.

Cooper J, Steeg S, Bennewith O, et al. (2013) Are hospital services for self-harm getting better? An observational study examining management, service provision and temporal trends in England. BMJ Open 3: e003444.

Currier GW, Fisher SG and Caine ED (2010) Mobile crisis team intervention to enhance linkage of discharged suicidal emergency department patients to outpatient psychiatric services: a randomized controlled trial. Academic Emergency Medicine, 17(1): 36-43.

Flannery F, Adams D and O'Connor N (2011) A community mental health service delivery model: integrating the evidence base within existing clinical models. Australasian Psychiatry 19(1): 49-55.

Gunnell D, Bennewith O, Peters TJ, et al. (2005) The epidemiology and management of self-harm amongst adults in England. Journal of Public Health 27(1): 67-73.

Gunnell D, Hawton K, Ho D, et al. (2008) Hospital admissions for self harm after discharge from psychiatric inpatient care: cohort study. British Medical Journal, 337: a2278.

Harris EC and Barraclough B (1997) Suicide as an outcome for mental disorders. A meta-analysis. British Journal of Psychiatry 170: 205-228.

Haw C, Hawton K, Houston K, et al. (2001) Psychiatric and personality disorders in deliberate self-harm patients. The British Journal of Psychiatry 178(1): 48-54.

Hawton K and van Heeringen K (2009) Suicide. The Lancet 373(9672): 1372-1381.

Hawton K, Bergen H, Kapur N, et al. (2012) Repetition of self-harm and suicide following self-harm in children and adolescents: findings from the Multicentre Study of Self-harm in England. Journal of Child Psychology and Psychiatry and Allied Disciplines, 53(12), 1219-1219.

Kapur N, Steeg S, Turnbull P, et al. (2015) Hospital management of suicidal behaviour and subsequent mortality: a prospective cohort study. The Lancet Psychiatry, 2(9), 809-816.

Krysinska, K., Batterham, P. J., Tye, M., et al (2016) Best strategies for reducing the suicide rate in Australia. Australian and New Zealand Journal of Psychiatry, 50(2), 115-118.

Large M, Sharma S, Cannon E, et al. (2011) Risk factors for suicide within a year of discharge from psychiatric 
hospital: a systematic meta-analysis. Australian and New Zealand Journal of Psychiatry, 45(8): 619-628.

Larkin GL and Beautrais AL (2010) Emergency departments are underutilized sites for suicide prevention. Crisis 31(1): 1-6.

Mehlum L, Jørgensen T, Diep LM, et al. (2010) Is organizational change associated with increased rates of readmission to general hospital in suicide attempters? A 10-year prospective catchment area study. Archives of Suicide Research 14(2): 171-181.

Murphy E, Kapur N, Webb R, et al. (2012) Risk factors for repetition and suicide following self-harm in older adults: multicentre cohort study. British Journal of Psychiatry, 200(5), 399-404.

National Institute for Health and Clinical Excellence (2004) Self-harm: The short-term physical and psychological management and secondary prevention of self-harm in primary and secondary care. NHS.

Nock MK, Borges G, Bromet EJ, et al. (2008) Cross-national prevalence and risk factors for suicidal ideation, plans and attempts. British Journal of Psychiatry 192(2): 98-105.

Olfson M, Marcus SC and Bridge JA (2012) Emergency treatment of deliberate self-harm. Archives of General Psychiatry 69(1): 80-88.

Owens D, Horrocks J and House A (2002) Fatal and non-fatal repetition of self-harm. Systematic review. British Journal of Psychiatry 181(3): 193-199.

Schaffer A, Sinyor M, Kurdyak P, et al. (2016) Population based analysis of health care contacts among suicide descedants: identifying opportunities for more targeted suicide prevention strategies. World Psychiatry 15(2): 135-145.

Singhal A, Ross J, Seminog O, et al. (2014) Risk of self-harm and suicide in people with specific psychiatric and physical disorders: comparisons between disorders using English national record linkage. Journal of the Royal Society of Medicine, 107(5): 194-204.

StataCorp (2013) Stata: Release 13.1. College Station TX: StataCorp LP.

Steeg S, Kapur N, Webb R, et al. (2012) The development of a population-level clinical screening tool for selfharm repetition and suicide: the ReACT Self-Harm Rule. Psychological Medicine 42(11): 2383-2394.

Suominen K, Isometsä E, Martunnen M, et al. (2004) Health care contacts before and after attempted suicide among adolescent and young adult versus older suicide attempters. Psychological Medicine 34(2): 313321.

Suominen KH, Isometsä ET, Ostamo AI, et al. (2002) Health care contacts before and after attempted suicide. Social Psychiatry and Psychiatric Epidemiology 37(2): 89-94. 
Table 1: Characteristics of the 42,353 individuals admitted to hospital for for self-harm, NSW $2005-2011$

\begin{tabular}{|c|c|c|}
\hline Characteristic & $\mathbf{n}$ & $\%$ \\
\hline \multicolumn{3}{|l|}{ Length of stay in hospital } \\
\hline 1 day or less & 25,108 & 59.3 \\
\hline 2-4 days & 9,307 & 22.0 \\
\hline 5-9 days & 3,903 & 9.2 \\
\hline 10-19 days & 2,184 & 6.2 \\
\hline 20-39 days & 775 & 1.8 \\
\hline 30 days or more & 1,076 & 2.5 \\
\hline Admission for self harm in the previous 2 years & 1,295 & 3.1 \\
\hline Admitted to a psychiatric ward & 3,061 & 7.2 \\
\hline \multicolumn{3}{|l|}{ Age at admission } \\
\hline $15-19$ & 7,016 & 16.6 \\
\hline $20-29$ & 10,480 & 24.7 \\
\hline 30-39 & 9,374 & 22.1 \\
\hline $40-49$ & 7,771 & 18.4 \\
\hline $50-59$ & 4,267 & 10.1 \\
\hline 60 and over & 3,445 & 8.1 \\
\hline Female & 24,155 & 57.0 \\
\hline \multicolumn{3}{|l|}{ Relationship status at admission } \\
\hline Partnered & 11,519 & 27.2 \\
\hline Single & 28,638 & 67.6 \\
\hline Unknown & 2,196 & 5.2 \\
\hline \multicolumn{3}{|l|}{ Method } \\
\hline Poisoning & 32,117 & 75.8 \\
\hline Cutting/piercing & 6,808 & 16.1 \\
\hline Hanging & 1,160 & 2.8 \\
\hline Motor vehicle exhaust & 625 & 1.5 \\
\hline Jumping & 268 & 0.6 \\
\hline Other & 1,374 & 3.2 \\
\hline Any psychiatric disorder & 31,489 & 74.3 \\
\hline Substance misuse disorder & 12,294 & 29.0 \\
\hline Schizophrenia and related psychoses & 2,391 & 5.7 \\
\hline Mania & 1,806 & 4.3 \\
\hline Depression & 15,726 & 37.1 \\
\hline Anxiety disorder & 10,086 & 23.8 \\
\hline Eating disorder & 381 & 0.9 \\
\hline Personality disorder & 3,906 & 9.2 \\
\hline
\end{tabular}

Note: the psychiatric disorders are not mutually exclusive: a patient can have a diagnosis of more than one disorder. As such, the sum of the counts in these categories is greater than the sample size. 
Table 2: Receiving community mental health care within 30 days of discharge for patients connected and unconnected with community mental health services

\begin{tabular}{|l|r|r|}
\hline & \multicolumn{2}{|c|}{ Community mental health care } \\
\hline $\begin{array}{l}\text { Connected with community } \\
\text { mental health services }\end{array}$ & No & Yes \\
\hline Unconnected & $20,131(72.9 \%)$ & $7,487(27.1 \%)$ \\
\hline Connected & $5,055(34.3 \%)$ & $9,680(65.7 \%)$ \\
\hline
\end{tabular}


Table 3: Predictors of community mental health care within 30 days of discharge for self harm

\begin{tabular}{|c|c|c|}
\hline Characteristic & $\begin{array}{l}\text { Adjusted Odds Ratio } \\
(95 \% \text { CI })\end{array}$ & p-value \\
\hline $\begin{array}{l}\text { Connected to community mental health services (vs. } \\
\text { unconnected) }\end{array}$ & 5.33 (5.09 to 5.59) & $<0.0001$ \\
\hline Length of stay in hospital & & $<0.0001$ \\
\hline 1 day or less (ref.) & 1.00 & \\
\hline 2-5 days & $0.83(0.78$ to 0.88$)$ & \\
\hline 6-9 days & $0.81(0.75$ to 0.88$)$ & \\
\hline 10-19 days & $0.91(0.82$ to 1.01$)$ & \\
\hline 20-39 days & $1.01(0.85$ to 1.19$)$ & \\
\hline 30 days or more & $0.87(0.75$ to 1.01$)$ & \\
\hline $\begin{array}{l}\text { Admission for self-harm in previous } 2 \text { years (vs. no previous } \\
\text { admission) }\end{array}$ & $0.91(0.80$ to 1.03$)$ & 0.15 \\
\hline Admitted to a psychiatric ward (vs. general admission) & $0.88(0.81$ to 0.97$)$ & 0.008 \\
\hline Age at admission & & $<0.0001$ \\
\hline $15-19$ (ref.) & 1.00 & \\
\hline $20-29$ & $0.83(0.77$ to 0.89$)$ & \\
\hline $30-39$ & $0.81(0.75$ to 0.87$)$ & \\
\hline $40-49$ & $0.84(0.78$ to 0.91$)$ & \\
\hline $50-59$ & $0.81(0.74$ to 0.89$)$ & \\
\hline 60 and over & $0.70(0.63$ to 0.77$)$ & \\
\hline Female (vs. male) & 1.07 (1.02 to 1.12$)$ & 0.005 \\
\hline Single (vs. in relationship) & $0.97(0.92$ to 1.02$)$ & 0.28 \\
\hline \multicolumn{3}{|l|}{ Method } \\
\hline Poisoning (ref.) & 1.00 & $<0.0001$ \\
\hline Cutting/piercing & $0.96(0.90$ to 1.02$)$ & \\
\hline Hanging & 1.27 (1.11 to 1.45$)$ & \\
\hline Motor vehicle exhaust gas & 1.30 (1.08 to 1.55$)$ & \\
\hline Jumping & $0.71(0.53$ to 0.95$)$ & \\
\hline Other & $0.82(0.72$ to 0.93$)$ & \\
\hline Substance misuse disorder (vs. no diagnosis) & $0.78(0,74$ to 0.82$)$ & $<0.0001$ \\
\hline Schizophrenia and related psychoses (vs. no diagnosis) & $1.46(1.32$ to 1.61$)$ & $<0.0001$ \\
\hline Mania (vs. no diagnosis) & $1.11(1.00$ to 1.24$)$ & 0.055 \\
\hline Depression (vs. no diagnosis) & $1.24(1.18$ to 1.30$)$ & $<0.0001$ \\
\hline Anxiety disorder (vs. no diagnosis) & $1.15(1.10$ to 1.21$)$ & $<0.0001$ \\
\hline Eating disorder (vs. no diagnosis) & $0.92(0.74$ to 1.16$)$ & 0.49 \\
\hline Personality disorder (vs. no diagnosis) & $0.90(0.84$ to 0.97$)$ & 0.008 \\
\hline
\end{tabular}




\section{University Library}

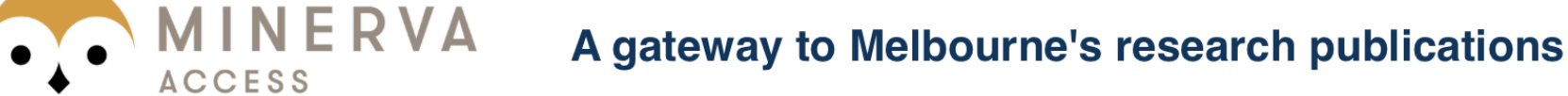

Minerva Access is the Institutional Repository of The University of Melbourne

Author/s:

Spittal, MJ;Shand, F;Christensen, H;Brophy, L;Pirkis, J

Title:

Community mental health care after self-harm: A retrospective cohort study

Date:

2017-07-01

Citation:

Spittal, M. J., Shand, F., Christensen, H., Brophy, L. \& Pirkis, J. (2017). Community mental health care after self-harm: A retrospective cohort study. AUSTRALIAN AND NEW ZEALAND JOURNAL OF PSYCHIATRY, 51 (7), pp.727-735. https:// doi.org/10.1177/0004867416676366.

Persistent Link:

http://hdl.handle.net/11343/122842 